\title{
Advances in Multidimensional Synthetic Aperture Radar Signal Processing
}

\author{
Carlos López-Martínez, ${ }^{1}$ Laurent Ferro-Famil, ${ }^{2}$ and Andreas Reigber ${ }^{3}$ \\ ${ }^{1}$ Department of Signal Theory and Communications, Universitat Politècnica de Catalunya (UPC), c/Jordi Girona 1-3, \\ 08034 Barcelona, Spain \\ ${ }^{2}$ Institute of Electronics and Telecommunications of Rennes, University of Rennes 1, 263 Avenue Gènèral Leclerc, \\ 35042 Rennes Cedex, France \\ ${ }^{3}$ Microwaves and Radar Institute, German Aerospace Center (DLR), P.O. Box 1116, 82230 Weßling, Germany
}

Correspondence should be addressed to Carlos López-Martínez, carlos.lopez@tsc.upc.edu

Received 8 April 2010; Accepted 8 April 2010

Copyright (c) 2010 Carlos López-Martínez et al. This is an open access article distributed under the Creative Commons Attribution License, which permits unrestricted use, distribution, and reproduction in any medium, provided the original work is properly cited.

Synthetic Aperture Radar can be considered nowadays as an established and mature technology to obtain high-resolution two-dimensional reflectivity images of the Earth surface in nearly all weather conditions and independently of the day-night cycle. During a first period, ranging from their conception in the 1950s to the beginning of the 1990s, SAR systems were characterized by a single acquisition channel, and it was proven that this technology allows the observation and characterization of the Earth surface in the microwave region of the electromagnetic spectra. At the beginning of the 1990s, SAR technology started to show its important potential with the availability of multichannel or multidimensional SAR system configurations. Among them, it is worth to mention: interferometric techniques (InSAR), using single- or multiple baselines configurations, polarimetric diversity (PolSAR), or the combination of these approaches called polarimetric SAR interferometry (PolInSAR). It is also important to note that in the recent years, the use of time diversity, in combination with any of the previously introduced multidimensional configurations, has emerged as a new and promising research field to improve the analysis of the areas being imaged, but also the observation and characterization of dynamic processes. The importance and interest on the different multidimensional SAR system configurations is partly due to the increase of the number of radar observables but primarily to the fact that the acquired multidimensional signals are sensitive to different biophysical and geophysical properties of the Earth surface and may then be used for quantitative retrieval purposes.
Multidimensional SAR data have been shown to be very useful for the monitoring of the Earth surface in a wide range of applications. The spatial diversity exploited in InSAR configurations makes the exploration of the vertical structure of volumetric scenes possible. The complex correlation coefficient, constructed from two complex SAR images, represents here the most important radar observable. In the last 20 years, the sensitivity of the phase component to the terrain topography has been extensively exploited. The most relevant examples are the STRM mission, which provided, for the first time, the Earth topography at an almost global scale and the future Tandem-X mission, that pursues the same objective, but with an improved accuracy. InSAR has also demonstrated an enormous potential for the monitoring of terrain displacements using differential techniques. The amplitude of the complex correlation coefficient revealed to be very useful in diverse applications, like, for instance, volcano's pyroclastic flows monitoring, study and retrieval of stem volume over forested areas, monitoring of dry and wet snow covered areas, as well as for the analysis of ice covered rivers, and so forth. SAR systems exploiting polarization diversity permit to retrieve some of the geometrical and scattering properties of the targets being imaged. One of the first applications of PolSAR data has been terrain classification, for which different procedures have been presented: unsupervised schemes based on incoherent decompositions, either supervised or unsupervised statistical classifications or techniques dedicated to particular scenarios, such as urban areas, for instance. Since the polarization 
orientation angle is geometrically related to topographical slopes and the radar look angle, PolSAR data may be also considered for the extraction of topographic information or the measurement of the ocean surface slopes. Polarimetric decomposition theorems play an important role in PolSAR remote sensing, since they provide an interpretation of the scattering phenomenon that may be used to isolate relevant physical information. PolSAR data have also succesfully been applied to the estimation of soils moisture and roughness. Polarimetric optimization procedures were shown to play a fundamental role for the quantitative extraction of forest and crop parameters, in the special case of PolInSAR, as well as for the improvement of differential techniques for the retrieval of terrain subsidence.

As the reader may see, the key and common point between all the multidimensional SAR configurations concerns the possibility to exploit the correlation structure that characterizes a set of jointly acquired SAR images, leading to the applications that indicated above. The fifteen manuscripts of this special issue cover all the different aspects of the exploitation of multidimensional SAR data for remote sensing purposes, from the basics concerned with data modeling and parameter estimation to advanced applications.

Multidimensional SAR data, especially when natural environments are imaged, are affected by speckle, which needs to be considered as a stochastic component. Its correct modeling has a critical impact on the final capability to retrieve geophysical and biophysical information. In the paper by A. Paul Doulgeris and T. Eltoft, the authors propose a novel framework to model PolSAR data based on a Scale Mixture of Gaussian Models. This approach permits, as demonstrated with experimental PolSAR data, to model non-Gaussian scenarios. These models present also the advantage to correctly account for Gaussian scenarios in case of stationary areas. After modeling the speckle components of multidimensional SAR data, it is necessary to filter it, without affecting neither the useful information of the multidimensional data nor the signal morphology. In the paper by L. Hongga et al., the authors introduce a technique addressing speckle noise reduction based on the theory of level sets. This method presents a big potential as it induces filtering based on a local analysis of the signal morphology and does not restrict filtering to a reduced set of spatial shapes.

As indicated previously, one of the essential characteristics of multidimensional SAR data resides in the large number of available radar observables that make a better characterization of the observed terrain possible. Consequently, a better classification or segmentation of the data can be achieved. In this sense, three contributions to this special issue address this topic. In the paper by L. Zhang et al., the authors focus on PolSAR data classification using Support Vector Machine techniques in which they do not directly consider PolSAR data, but rather the different parameters derived from polarimetric incoherent decompositions. The paper shows the key role of the different decomposition techniques when exploiting PolSAR data. In a second work, T. Zou et al. propose first a review and comparison of the different parameters and techniques that may be considered for the classification of PolSAR data. Finally, the authors introduce Extremely Randomized Clustering Forests techniques for PolSAR image classification comparing it with other competitive classifiers. Finally, in the paper by Y. Shuai et al., the authors address the problem of PolSAR data segmentation and present a level set-based method for object segmentation in PolSAR images. The authors propose a modified energy functional via active contour models based on complex Gaussian and Wishart distribution models for both single- and multilook PolSAR images segmentation.

Multidimensional SAR data can be acquired from spaceborne, air-borne or ground-based platforms. In the paper by M. Watanabe et al., PolSAR data acquired from an airborne and a ground-based systems are analyzed to monitor flooding in urban environments. The authors study simultaneously the different polarimetric scattering mechanisms that appear in each scenario. As the authors indicate, the fusion of data acquired from different platforms possesses a big potential for a better characterization of the scattering phenomena. This idea of data fusion is also pursued by C. He et al., by considering the joint use of PolSAR data with optical data within a multichannel image fusion algorithm based on a turbo iterative method. This paper clearly shows that an improved characterization of the terrain may be obtained by considering the fusion of data originated by sensors of different nature, as they operate over different regions of the electromagnetic spectra.

Other sources of signal diversity may be considered for a better characterization of the target under study. A problem that takes a clear advantage of the multidimensional SAR data nature is the detection of moving targets, addressed by four contributions. In a first paper, contributed by B. Cai et al., the authors propose and compare different Constant False Alarm Ratio detectors of moving targets based on along-track interferometry. A similar problem is studied in a second contribution by G. Ginolhac et al. Here, the authors consider also multistatic configurations, by assuming one transmitter and several receivers. The main contribution of this paper to the special issue is the enlargement of measurements to multistatic configurations beyond the traditional monostatic case, where the transmitter and the receiver are located at the same position. The third contribution addressing the moving target indicator problem, by $\mathrm{D}$. Wu et al., concerns the detection of moving targets in heterogeneous scenarios. As it is demonstrated by the authors, the inclusion of additional or prior information may improve the detection of targets. The detection of moving targets has become an important application in the recent years. This importance has crystallized into the fact that current and future SAR systems may consider an imaging mode specialized in the detection of moving targets. In the paper by S. Chui and M. Dragosevic, the authors analyzed the moving target indicator imaging mode of the Canadian Radarsat-2 system.

The first step that any SAR data have to undergo is the focusing process where all the contributions of a particular target are collected and focused at its location in the SAR image. In the paper by D. Flores-Tapia et al. the authors 
propose and validate a novel reconstruction algorithm for circular SAR. This novel imaging mode offers the possibility to look at the same target from different positions in space, that is, a source of diversity for new multidimensional SAR data. The possibility to obtain new sources of diversity is considered in the paper by L. Du et al., where the authors propose a new methodology for the reconstruction of threedimensional SAR images aiming to retrieve the vertical structure of a given target. SAR focusing techniques can be also considered for the through-wall imaging problem. In the paper by $\mathrm{Y}$. Hu et al., the authors propose the use of the the location inverse solution algorithm to reduce the ghost images in comparison with the backward projection algorithm.

SAR systems are inherently multifrequency systems, as they use frequency-modulated pulses to obtain a high range resolution. In the paper by D. Garmatyuk and J. Schuerger, the authors introduce a new alternative for an ultrawideband OFDM pulse model that presents clear advantages with respect to traditional schemes in presence of jamming.

We would like to finish this Editorial by, first of all, showing our appreciation to all the authors for submitting their contributions to this special issue. In addition, we would like also to extend our appreciation to all the reviewers who accepted to participate to the review process of the different works that were submitted to this special issue. Finally, we would also like to thank the staff from Hindawi for offering us the possibility to create this special issue.

Carlos López-Martínez Laurent Ferro-Famil Andreas Reigber 